\title{
Evaluation of MicroRNA-210 (miR-210) as a Diagnostic and Prognostic Biomarker in Pre-eclampsia Pregnancies
}

\begin{abstract}
Fetnat M. Tolba ${ }^{\mathrm{a}}$, Adel M. Amira M. Abdelrahman ${ }^{\text {a }}$

a

Department of clinical and chemical pathology, Faculty of Medicine, Benha university, Egypt. b Department of Gynecology and Obstetric, Faculty of Medicine, Benha university, Egypt.
\end{abstract}

Correspondence to: Maha A. Abdella, department of clinical and chemical pathology, Faculty of Medicine, Benha university, Egypt.

Email:

.mahaadel2020@gmail.com

Received: 15 September 2020

Accepted: 5 October 2020

\begin{abstract}
:
Objective: This study aims to assess the level of miR-210 in Egyptian women with pre-eclampsia (PE) and to evaluate its role in diagnosis and prognosis of the disease. Subjects and Methods: The study was conducted on 30 pregnant women with PE divided into two groups: group (I): included 15 cases with mild PE and group (II): included 15 cases of severe PE and, 20 healthy pregnant women with matched age and sex were included as control group. All women included in the study were subjected to, history taking full clinical examination, laboratory investigations included (CBC), (PT), (ALT, AST), (urea, creatinine), detection of protein in urine, as well as miRNA-210 gene expression by RTPCR. Results: Patients with PE showed a highly significantly increase in serum miR-210 ( $\mathrm{P}$ value $<0.001)$ compared to control as well as, it was higher in severe PE than in mild PE ( $\mathrm{P}$ value <0.001). MiR 210 have highly significant positive correlation with (systolic, diastolic, MABP), (Proteinuria) and (PT) a significant
\end{abstract} positive correlation with (AST), (ALT),.However no significant correlation was found with $(\mathrm{Hb} \%)$, platelet count, PTT and INR, serum urea and creatinine. PTT was statistically significant (P value 0.002).The best cutoff value of PE (2.03) with sensitivity and specificity of $90.0 \%$ and 85.0\% respectively. Conclusion: Expression of mir-210 is upregulated in pre-eclampsia and was higher in severe than in mild. Hence, the serum miR-210 can be used as a diagnostic, prognostic biomarker in PE patients and understanding pathophysiology.

Key Words: Pre-eclampsia , miR-210, RT_PCR 



\section{Introduction}

Pre-eclampsia is a multi-system disorder that is unique to human pregnancy, affecting $2-5 \%$ of pregnancies worldwide and leads to maternal deaths in 10-15\% (1).

Pre-eclampsia can manifest as either a maternal syndrome (hypertension and proteinuria with / without other multisystem dysfunction) or fetal syndrome.(2).

Although the etiology of pre-eclampsia is thought to be related to insufficient spiral arterial conversion leading to decreased uteroplacental perfusion and, therefore, is thought to give rise to chronic placental ischemia and hypoxia (3).

MicroRNAs are members of a larger class of non-coding RNAs that control gene expression and regulate biological processes by targeting mRNA and inducing translational repression or RNA degradation. MicroRNAs are on average a 70nt-long transcript (4). MicroRNAs expression affects several human cellular function from proliferation and differentiation to apoptosis and associating with miRNAs pathological alteration in diseases from cancer to myocardial infarction (5). miR-210 is associated with a variety of important processes like cell cycle regulation, cell survival, differentiation, angiogenesis, as in metabolism as in hypoxic conditions and cancer (6),(7).

Over expression of miR-210 can regulate cell response to hypoxia through mitochondrial function promoting the shift from mitochondria respiration to glycolysis and induce angiogenesis (8). Also in placental tissue isolated from pre-eclamptic patients shows miR-210 expression is increased (9).

Previous studies have demonstrated that the dysregulation of the specific plasma-related miRNAs expression is associated with the development of PE (1) and (10).

\section{Aim of the study}

This study aimed to assess the level of miR210 expression in the serum of Egyptian women with pre-eclampsia and to evaluate its role in diagnosis and prognosis of PE.

\section{Subjects}

This case/control study was conducted on 30 pregnant women with PE divided into two groups: group (I): included 15 cases with mild PE, group (II): included 15 cases of severe PE .In addition, 20 healthy pregnant women with matched age and sex representing the control group (III). 
PE patients were admitted to Gynecology and Obstetrics Department in Benha University Hospitals. The study was conducted from the period of (January 2018 to January 2019.) after approval of the local ethical committee of Benha University. Informed written consent was obtained from each participant.

PE patients was classified According to a study done in 2018:, mild PE was diagnosed when blood pressure $\geq 140 / 90 \mathrm{mmHg}$ on 2 occasions, at least 6 hours apart, but without evidence of end-organ damage, in a woman who was normotensive before 20 weeks' gestation, and the systolic blood pressure (SBP) increased by $30 \mathrm{mmHg}$ or diastolic blood pressure (DBP) increased by 15 $\mathrm{mmHg}$. Positive proteinuria after 20 weeks of gestation.

Severe PE was diagnosed when SBP of $\geq 160 \mathrm{mmHg}$ or DBP of $\geq 110 \mathrm{mmHg}$ on 2 occasions at least 6 hours apart in a normotensive pregnant woman before pregnancy. Proteinuria of more than $(5 \mathrm{~g})$ in a 24-hour collection And Presence of symptoms of severe PE were detected such as pulmonary edema, cyanosis, oliguria and/or impaired liver function, thrombocytopenia, oligohydramnios, decreased fetal growth, or placental abruption.(11).

Exclusion criteria:

- Fetal congenital malformations or chromosomal abnormalities.

- Recent infection.

- Anti-phospholipid syndrome.

- Trauma.

- Drug or alcohol abuse.

- Preexisting hypertension.

- Thrombophilia with history of PE.

- Receiving anticoagulant.

\section{Methods}

All cases included in the study were subjected to:

1- Full history taking: such as personal and maternal history including: age, gravidity, positive family history, gestational age and onset of preeclampsia.

2- Clinical examination: General and clinical examination including: BP, Mean Arterial Pressure (MABP), edema, weight, height, and body mass index (BMI). 
3- Laboratory investigation:

\section{Blood sample:}

- 6 mL of venous blood was drawn under complete aseptic conditions:

- $\mathrm{CBC}$ by $1 \mathrm{~mL}$ blood was mixed with EDTA used, by automated cell counter (Sysmex KX 21N; Sysmex, Inc., Mundelein, IL, USA).

- Coagulation profile (PT, PTT, INR) by $1.6 \mathrm{ml}$ was added on ( Na citrated tube for) using automated coagulometer (Coatron A4, Teco, Germany).

- The rest of blood was withdrawn into plain tube and separated serum was aliquoted and stored at $-80^{\circ} \mathrm{C}$ until used. The stored serum then thawed and used for:

1. Biochemical assays: liver function tests and kidney function tests using Biosystem A15 auto-analyzer (Biosystems S.A., Barcelona, Barcelona, Spain).

2. miRNA-210 detection by RT-PCR.

Urine sample: $24 \mathrm{~h}$ Urine sample was collected \&centrifuged for protein estimation by a turbidimetric method (using TCA 3\%, by Biosystem reagent kit using semi-automated analyzer BTS-350 (Biosystem S.A., Barcelona, Spain).)

- Methods of miRNA-210 expression level by RT- PCR.

1-Purification of total RNA, including miRNA(Extraction).

QIAzolLysis Reagent was used to extract total RNA including microRNA from samples using miRNeasy Mini Kit (cat. no. 217004) (Qiagen,Germany) according to the manufacturer's protocol. QIAzolLysis Reagent was added to serum samples to facilitate lysis. After addition of chloroform, the lysate was separated in to aqueous and organic phases by centrifugation. RNA partitions were at the upper aqueous phase, RNA was extracted, and ethanol was added to provide suitable binding conditions for all RNA molecules. High-quality RNA was eluted in a small volume of RNase-freewater.

Detection of RNA purity: by Nanodrop 2000 spectrophotometer (USA).

The ratio of absorbance at $260 \mathrm{~nm}$ and 280 $\mathrm{nm}$ is used to assess the purity of RNA. A ratio of nearly 2.0 is generally accepted as pure for RNA. If it is lower, this indicates protein, phenol or other contaminants that absorb strongly at or near $280 \mathrm{~nm}$. It is 
affected by change in sample acidity, wavelength accuracy of spectrophotometer and nucleotide mix in the sample.

260/230 ratio: Secondary measure of nucleic acid purity. Expected values are in the range of 2.0-2.2 (12).

2- Reverse transcription for quantitative real-time PCR (1st step):

cDNA was produced using miScriptII RT Kit (Cat.no.217004 QIAGEN, Germany) according to manufacturer instructions. The reverse transcription master mix was prepared using miScript HiSpec Buffer, miScript Nucleics Mix and miScript Reverse Transcriptase Mix. MiScript HiSpec Buffer converts mature miRNAs and certain small nucleolar RNAs and small nuclear RNAs selectively in to cDNA. The volume of RNA was different in each sample due to different concentrations so to use equal amounts of total RNA, the samples were diluted in RNase-freewater. Template RNA and RNase-freewater $(12 \mu \mathrm{L})$ was added to each tube containing reverse transcription master $\operatorname{mix}(8 \mu \mathrm{L})$ forming final volume $20 \mu \mathrm{L}$ for each reaction in $0.2 \mathrm{~mL}$ polymerase chain reaction (PCR) tubes. Then mixed gently and briefly centrifuged. Tubes were then incubated at $37{ }^{\circ} \mathrm{C}$ for $60 \mathrm{~min}$. followed by incubation at $95{ }^{\circ} \mathrm{C}$ for 5 mins. In Veriti thermal cycler Applied Biosystem (USA).

3-Real-time PCR for detection of miRNA210 (2nd step):

Quantification of miRNA was performed using miScript SYBR Green PCR Kit supplied by (QIAGEN, Germany) (cat.no 217004) according to the manufacturer's protocol. Each reaction was performed in a final volume of $25 \mu \mathrm{l}$ holding $2.5 \mu \mathrm{l}$ of cDNA, $2.5 \mu \mathrm{l}$ of miScript universal primer, $2.5 \mu \mathrm{l}$ of miScript primer assay (RNU 6 or miRNA 210), $5 \mu \mathrm{l}$ of RNAse free water and12.5 $\mu \mathrm{l} \mathrm{SYBR} \mathrm{Green} \mathrm{PCR} \mathrm{Master} \mathrm{mix.}$ Forward and reverse miRNA specific primers were supplied by (Qiagen, Germany). MiR-210-5P primers, 5'AGCCCCUGCCCACCGCACACUG - $3^{\prime}$ and RNU6, 5'- CTCGCTTCGGCAGCACA (F) $-3^{\prime}$

Real-time PCR was performed on Real-time PCR cycler Applied Biosystems (USA) under the following conditions: Initial activation step at $95^{\circ} \mathrm{C}$ for $15 \mathrm{~min}$, followed by 40 cycles of denaturation at $94^{\circ} \mathrm{C}$ for $30 \mathrm{~s}$., annealing at $55^{\circ} \mathrm{C}$ for $1 \mathrm{~min}$. and extension at $70^{\circ} \mathrm{C}$ for 1 min., in which fluorescence was acquired. 
Normalization controls: the normalization control used was RNU6-2.

Analysis:

Detection and quantification of each gene was expressed as relative miRNA level compared with a standard housekeeping gene (RNU6-2) Selection of reference gene was done after evaluating its stability using the comparative delta-CT method (13).

The assessment of the obtained data from the miRNA expression was done by using the cycle threshold $(\mathrm{Ct})$ method. The cycle threshold $(\mathrm{Ct})$ is defined as the number of cycles required for the fluorescent signal to cross the threshold in RT-PCR. The expression level of the miRNA was reported as $\Delta \mathrm{Ct}$ value, the fold change in the expression level of the miRNA was calculated (fold change $=2-\Delta \Delta \mathrm{Ct}$ ) (13).

Statistical Methods

Data management and statistical analysis were done using SPSS vs.25. (IBM, Armonk, New York, United states).

Comparisons between groups were done using either Mann Whitney $U$ test; for numerical data, or Chi-square test; for categorical data. Mir210 level was compared between different degrees of severity and controls using Kruskal Wallis test. Post hoc analysis was done which were all Bonferroni adjusted.

Correlation analyses were done between Mir210 and other parameters using Spearman's correlation. "r",. Which ranges from -1 to +1 . -1 indicates strong negative correlation, while +1 indicates strong positive correlation, and 0 indicates no correlation.

ROC analysis was done for Mir210 in diagnosing preeclampsia. (AUC), the best cutoff point, diagnostic indices including sensitivity, specificity, PPV and NPV were calculated. All P values were two sided. P values less than 0.05 were considered significant.

\section{Results}

The general characteristics of the studied groups was shown in table (1) shows no significant difference between (PE patients) and (control groups) regarding their maternal age, gestational age, body mass index and parity state ( $\mathrm{p}$ value>0.05). Systolic, diastolic, mean arterial blood pressure), (AST),(Proteinuria) and (Mir210)were highly significant increased in (PE patients) and (ALT)was significantly 
increased than controls with $\mathrm{P}$ value $(<0.05)$

Table (1):Comparison of demographic and laboratory data between the studied groups.

\begin{tabular}{|c|c|c|c|c|}
\hline & & $\operatorname{Cases}(n=30)$ & Controls $(\mathbf{n}=20)$ & $P$ value \\
\hline Maternal age (Years) & Mean \pm SD & $29 \pm 4$ & $28 \pm 3$ & 0.305 \\
\hline Gestational age (Wks) & Mean \pm SD & $30 \pm 5$ & $33 \pm 3$ & 0.243 \\
\hline Body Mass Index & Mean \pm SD & $33.96 \pm 4.22$ & $31.66 \pm 6.28$ & 0.096 \\
\hline \multirow[t]{2}{*}{ Parity state } & PG n $(\%)$ & $23(76.7)$ & $16(80.0)$ & 0.780 \\
\hline & MG n (\%) & $7(23.3)$ & $4(20.0)$ & \\
\hline SBP (mmHg) & Mean \pm SD & $163 \pm 22$ & $112 \pm 10$ & $<0.001$ \\
\hline DBP (mmHg) & Mean \pm SD & $108 \pm 11$ & $76 \pm 6$ & $<0.001$ \\
\hline MABP (mmHg) & Mean \pm SD & $126 \pm 14$ & $87 \pm 7$ & $<0.001$ \\
\hline Hemoglobin (gm/dl) & Mean \pm SD & $10.1 \pm 1.5$ & $10.3 \pm 1.3$ & 0.677 \\
\hline Platelets(1000/ul) & Mean \pm SD & $199 \pm 55$ & $206 \pm 37$ & 0.422 \\
\hline PT (Sec) & Mean \pm SD & $13.94 \pm 2.82$ & $13.54 \pm 1.47$ & 0.812 \\
\hline INR & Mean \pm SD & $1.19 \pm 0.23$ & $1.12 \pm 0.12$ & 0.26 \\
\hline PTT (Sec) & Mean \pm SD & $31 \pm 4.3$ & $35.9 \pm 5.2$ & 0.002 \\
\hline Urea $(\mathbf{m g} / \mathbf{d l})$ & Mean \pm SD & $42 \pm 15$ & $39 \pm 16$ & 0.307 \\
\hline Creatinine (mg/dl) & Mean \pm SD & $1.02 \pm 0.33$ & $0.93 \pm 0.31$ & 0.364 \\
\hline $\operatorname{AST}(\mathbf{U} / L)$ & Median (range) & $37(11-300)$ & $24(12-112)$ & 0.003 \\
\hline $\operatorname{ALT}(\mathbf{U} / \mathbf{L})$ & Median (range) & $52(11-230)$ & $36(27-139)$ & 0.025 \\
\hline Proteinuria (mg/24h) & Median (range) & $1802(341-10863)$ & $89(43-143)$ & $<0.001$ \\
\hline Mir210 -5p (fold) & Median (range) & $6.01(1.62-18.62)$ & $1.17(1-6.91)$ & $<0.001$ \\
\hline
\end{tabular}

Mann Whitney $U$ test was used for numerical data. Chi-square test was used for categorical data. $(\mathrm{P}<0.05)$ was statistically significant and $(\mathrm{P}<0.005)$ as highly significant.

The expression level of Mir210 was highly significant increase in PE patients compared to control group $(\mathrm{P}<0.001)$. MiR-210 was significantly higher in patients with PE than controls (P value : < 0.001).It significantly higher in severe PE compared to patients with mild PE (P value : < 0.001) as shown in table (2) . Mir-210 showed a statistically highly significant correlation (positive correlation with systolic, diastolic and mean 
arterial blood pressure, PT and Proteinuria) according to table (3) . ROC analysis was done for using Mir210 to diagnose preeclampsia. It revealed a significant Area Under Curve (AUC) of 0.933 with $95 \%$ confidence interval ranged from 0.863 to
1.0. The best cutoff value for diagnosis of PE was 2.03 with sensitivity and specificity of $90.0 \%$ and $85.0 \%$ respectively and PPV \& NPV of $90.0 \%$ and $85.0 \%$ respectively. P value was $(<0.001)$ as in figure $(2)$.

Table (2): Mir210 level in all studied groups:

\begin{tabular}{lccccccc}
\hline & \multicolumn{2}{c}{ Severe } & \multicolumn{2}{c}{ Mild } & \multicolumn{2}{c}{ Controls } & \multirow{2}{*}{ P value } \\
& Mean & \pm SD & Mean & \pm SD & Mean & \pm SD & \\
\hline \multirow{2}{*}{ Mir210 (fold) } & 11.52 & 3.68 & 3.47 & 1.17 & 1.83 & 1.51 & $<0.001$ \\
\hline
\end{tabular}

Kruskal Wallis test was used. Post hoc analysis was done and different letters indicate significant pair . All post hoc were Bonferroni adjusted.

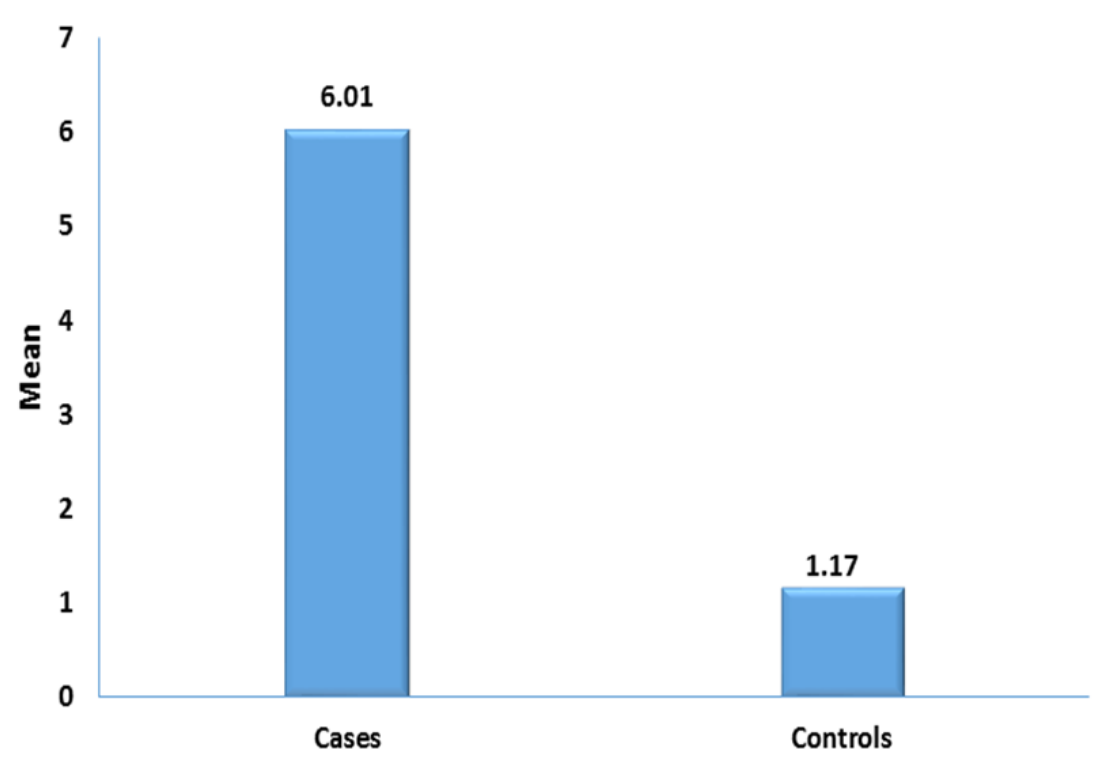

Figure (1):Mir-210 in both groups 
Benha medical journal vol. 38, issue 1, 2021

Table (3): Correlation coefficient (r) between Mir210 and the study parameters

\begin{tabular}{lll}
\hline Mir210 & r & P value \\
\hline Maternal age (Years) & 0.012 & 0.951 \\
Body Mass Index & 0.006 & 0.975 \\
Gestational age (Weeks) & -0.203 & 0.282 \\
Systolic blood pressure (mmHg) & $0.402^{*}$ & 0.027 \\
Diastolic blood pressure (mmHg) & $0.473^{* *}$ & 0.008 \\
Mean Arterial Pressure & $0.505^{* *}$ & 0.004 \\
Hemoglobin (gm/dl) & 0.269 & 0.151 \\
Platelets(1000/ul) & -0.017 & 0.928 \\
PT (Sec) & $0.493^{* *}$ & 0.006 \\
PTT (Sec) & -0.162 & 0.394 \\
INR & 0.262 & 0.162 \\
Urea (mg/dl) & -0.1612 & 0.396 \\
Creatinine (mg/dl) & -0.097 & 0.6122 \\
AST (U/L) & 0.214 & 0.256 \\
ALT (U/L) & 0.187 & 0.324 \\
Proteinuria(mg/24h) & $0.755^{* *}$ & $<.001$ \\
\hline
\end{tabular}

Spearman's correlation was used, $\mathrm{r}=$ Correlation coefficient, $*$ Significant, **highly significant.

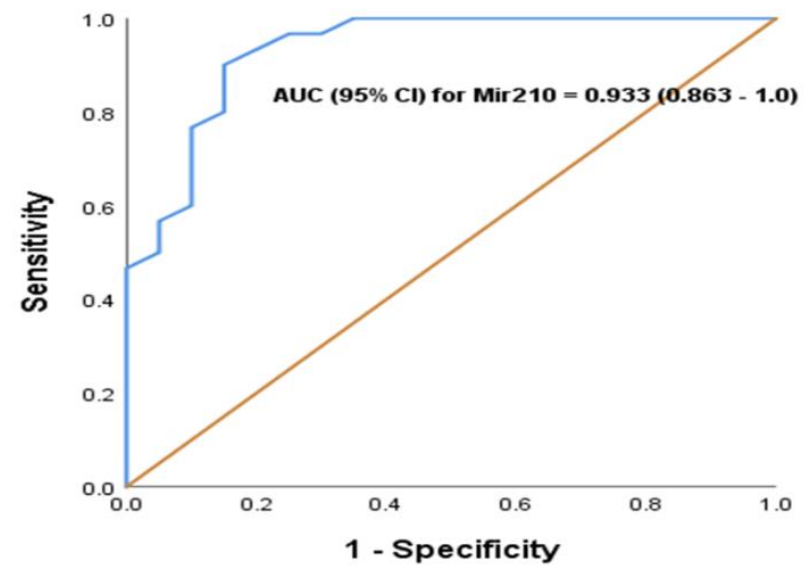

Figure (2): Roc curve of Mir210 in diagnosing pre-eclampsia 
Discussion

miR-210 was identified in placenta of pregnant women with $\mathrm{PE}$ that has been suggested to induce repression of trophoblast cell invasion (14).

As for laboratory data of the groups, there was no significant difference between patients and controls regarding hemoglobin level or platelet count $(\mathrm{P}$ value $=0.677 \&$ 0.422, respectively). The same was for PT and INR $(\mathrm{P}$ value $=0.812 \& \quad 0.26$, respectively), however, PTT was statistically significantly shorter in patients compared to controls, $(\mathrm{P}$ value $=0.002)$ and it was with in normal range.

PTT of PE patients compared with control group in the current study was not in agreement with other studies who observed that values of PTT were higher in the severe pre-eclamptic patients compared to controls (15) and16). In another study it was found that sPE patients had abnormal coagulation profile (17), it was explained by a certain degree of coagulative dysfunction which occurs in the endogenous coagulative pathways of sPE, while exogenous coagulative pathways do not change (18).

There was no significant difference between patients and controls regarding serum urea and creatinine $(\mathrm{P}$ value $=0.307,0.364$ respectively).But, ALT and proteinuria levels were highly significant increased and AST was significantly elevated in patients than controls $(\mathrm{P}$ value $=0.003,<0.001$, 0.025 respectively).

Other studies are agreed with our study noticed that significant increase in serum value of serum bilirubin and liver enzymes between preeclampsia cases compared to healthy pregnant, which explained by the effect of hypoxia of liver cells leading to necrosis of hepatocytes.(19) and (20), that increase was during the first 20 weeks of pregnancy which associated with higher risk for the development of severe preeclampsia in the second half of the pregnancy (21), that increased probability of maternal and fetal complications (22).

Regarding proteinuria, which was highly significantly elevated with PE in our study ( $\mathrm{P}$ value $<0.001$ ), in agreement with other studies, this elevation is due to reversible kidney damage (23 and 24).

As regards miR-210, we found higher expression level in preeclampsia patients compared to controls with a high significant value ( $\mathrm{P}$ value $<0.001)$.which agreed with 
other studies that confirming the role of elevated serum miR-210 as non-invasive method in diagnosis, prognosis and identifying women at-risk for monitoring and treatment through contribute to trophoblast function. (25) and (26). Other studies clarified that the expression levels of serum miR-210 was elevated starting from the second trimester in pregnancies complicated with PE.(1),(27)and(28)

Also other studies were similarly to our study reported that expression of placental miR-210 was up-regulated in patients with pre-eclampsia, due to inhibit the migration and invasion ability of trophoblast cells by placental miR-210, which regulated by transcriptional factor HIF-1 as well as NF«Bp50 leading to decreased STAT6and IL-4 .(29), (30) and (31).

The miR-210 expression may contribute to the occurrence of PE by interfering with potassium channel modulatory factor 1 mediated signaling in the human placenta. (32), which they studied possible regulatory mechanisms by system biology approaches Through identifying regulated genes related to $\mathrm{PE}$; targeted by human miR-210 .(33)

It was explained that high miR-210 expression is a possible modulator of mitochondrial dysfunction during PE. (34); and also observed that hypoxia markedly increased miR-210 expression in PE placenta. (35). However, miR-210 was among the32 up-regulated key microRNAs and genes in PE (36).

In the present study, miR-210 was highly significantly elevated in patients with severe PE compared to both patients with mild PE and to control group ( $\mathrm{P}$ value $>0.001$ ). These results suggested an association between miR-210 expression level and the severity of PE. In agreement of this study (37)

miR-210 in this study showed a significant positive correlation with SBP, DBP and MABP, PT as well as with proteinuria (P Value $=0.027,0.008,0.004,0.006$, and $<0.001$ respectively). In the same point of view, the urine miR-210 had a positive correlation with 24-hour urine proteins confirming the possibility of using urine miR-210 in evaluating the severity of kidney damage among PE patients. (1)

In addition, non-significant negative correlation was found in current study between mir210 and gestational age, platelet count, PTT, urea and creatinine level. (P value $=0.282,0.928,0.394,0.396 \& 0.6122$ respectively). Moreover, non-significant positive correlation was found between mir210 and maternal age, BMI, hemoglobin 
level, INR, AST\& ALT level ( $\mathrm{P}$ value = $0.931,0.975,0.151,0.162,0.256 \& 0.324$, respectively). ROC analysis was done for using mir210 for diagnosing preeclampsia. It revealed a significant AUC of 0.933 with $95 \%$ confidence, interval ranged from 0.863 to 1.0. miR-210 had sensitivity and specificity of $90.0 \%$ and $85.0 \%$ respectively with PPV and NPV of $90.0 \%$ and $85.0 \%$ respectively, which in agreement with the results of ROC curve were (AUC was 0.750 with $95 \%$ of confidence) (1).

\section{Conclusion}

Expression of mir-210 is upregulated in pregnant women with pre-eclampsia and it was found higher in severe than in mild preeclampsia. So, the serum miR-210 can be used as a non-invasive diagnostic and prognostic biomarker in pregnant women with pre-eclampsia. More studies with large sample size still needed to be investigated by increasing the sample size and its role in pathophysiologyof the PE.

Abbreviations:

APTT $=$ activated partial thromboplastin time,

$\mathrm{AUC}=$ area under the curve,

$\mathrm{PE}=$ pre-eclampsia,

$\mathrm{PT}=$ partial thromboplastin time,

miRNA $=$ microRNA,
HIF-1 =hypoxic inducible factor.

$\mathrm{NF}-\kappa \mathrm{B}=$ nuclear factor kappa $\mathrm{B}$

qRT-PCR = quantitative reverse transcriptasepolymerase chain reaction,

$\mathrm{ROC}=$ receiver operating characteristic.

* The authors declare that they have no conflict of interest.

\section{References}

1. Gan L, Liu Z, Wei M, Chen Y, Yang X, Chen L, et al,. MiR-210 and miR-155 as potential diagnostic markers for pre-eclampsia pregnancies. Medicine. 2017;96(28).

2. Mol BW, Roberts CT, Thangaratinam S, Magee LA, De Groot CJ and Hofmeyr GJ. Preeclampsia. The Lancet. 2016;387(10022):9991011.

3. Akinci $\mathrm{S}$, Ozcan $\mathrm{H}$, Balat $\mathrm{O}$, Ugur $\mathrm{MG}$, Ozturk E, Taysi S, et al,. Assessment of 8hydroxydeoxyguanosine levels in patients with preeclampsia: a prospective study. Clinical and experimental obstetrics \& gynecology. 2017;44(2):226-9.

4. Paul P, Chakraborty A, Sarkar D, Langthasa M, Rahman M, Bari M, et al,. Interplay between miRNAs and human diseases. Journal of cellular physiology. 2018;233(3):2007-18.

5. Rupaimoole R and Slack FJ. MicroRNA therapeutics: towards a new era for the management of cancer and other diseases. Nat Rev Drug Discov. 2017;16(3):203-222.

6. Ren CX, Leng RX, Fan YG, Pan HF, Wu $\mathrm{CH}$ and Ye DQ. MicroRNA-210 and its theranostic 
potential. Expert opinion on therapeutic targets. 2016;20(11):1325-38.

7. Xin Huang. Regulation of the hypoxic response by non-coding RNAs.Tumor Hypoxia:2017; p.189-224.

8. Zaccagnini G, Maimone B, Fuschi P, Maselli D, Spinetti G, Gaetano C, et al,. Overexpression of miR-210 and its significance in ischemic tissue damage. Scientific reports. 2017;7(1):1-0.

9. Korkes HA, Oliveira L De, Sass N, Salahuddin S, Karumanchi A and Rajakumar A. Relationship between hypoxia and downstream pathogenic pathways in preeclampsia HypertensGestational hypertension, preeclampsia and intrauterine growth restriction induce dysregulation of CVD \& CNS disease associated miRNAs in maternal whole peripheral blood.Thromb Res2017;137:126-40.

10. Hromadnikova I, Kotlabova K, Hympanova L and Krofta L. Hypertension, preeclampsia and intrauterine growth restriction induce dysregulation of cardiovascular and cerebrovascular disease associated microRNAs in maternal whole peripheral blood. Thromb Res2016;137: 126-40.

11. Brown, MA, Magee LA, Kenny LC, Karumanchi A, Mccarthy F, Saito $\mathrm{S}$ et al., Hypertensive disorders of pregnancy: ISSHP classification, diagnosis, and management recommendations for international practice. Hypertension. (2018). (72): 24-43.

12. Wilfinger $\mathrm{W}$, Mackey $\mathrm{K}$ and Chomczynski P. Effect of $\mathrm{pH}$ and ionic strength on the spectrophotometric assessment of nucleic acid purity. Biotechniques. 1997; 22(3):474-6, 478.

13. Livak KJ and Schmittgen TD. Analysis of Relative Gene Expression Data Using Real-Time Quantitative PCR and the 2- $\Delta \Delta \mathrm{CT}$ Method. Methods2001; (25): 402-408.

14. Koushki M, Amiri Dash Atan N, OmidiArdali $\mathrm{H}$ and RezaeiTavirani M. Assessment of Correlation Between miR-210 Expression and PreEclampsia Risk: A Meta-Analysis. Reports of biochemistry \& molecular biology2018; 7: 94-101.

15. Han L, Liu X, Li H, Zou J, Yang Z, Han J et al. Blood coagulation parameters and platelet indices: changes in normal and preeclamptic pregnancies and predictive values for preeclampsia. PLoS One2014; 9(12): e114488.

16. Jahromi BN and Rafiee SH. Coagulation factors in severe preeclampsia. Iranian Red Crescent Medical Journal2009; 11: 321-324.

17. Feldstein O, Kovo M, Tal O, Braunstein M, Grinstein E, Schreiber L, et al,. The association between abnormal coagulation testing in preeclampsia, adverse pregnancy outcomes, and placental histopathology.Hypertension in Pregnancy2019; 38: 176-183.

18. Szabo $G$ and Bala S. MicroRNAs in liver disease. Nat Rev Gastroenterol Hepatol. 2013; (10): 542-52.

19. Dacaj R, Izetbegovic S, Stojkanovic G and Dreshaj S. Elevated Liver Enzymes in Cases of Preeclampsia and Intrauterine Growth Restriction.Medical archives (Sarajevo, Bosnia and Herzegovina)2016; 70: 44-7. 
20. Munazza B, Raza N, Naureen A, Khan SA, Fatima F, Ayub M, et al,. Liver function tests in preeclampsia. Journal of Ayub Medical College, Abbottabad 2011; JAMC23: 3-5.

21. Mei-Dan E, Wiznitzer A, Sergienko R, Hallak $M$ and Sheiner E. Prediction of preeclampsia: liver function tests during the first 20 gestational weeks. The journal of maternal-fetal \& neonatal medicine: the official journal of the European Association of Perinatal Medicine, the Federation of Asia and Oceania Perinatal Societies, the International Society of Perinatal Obstetricians2013; 26: 250-3.

22. Thangaratinam S, Koopmans CM, Iyengar S, Zamora J, Ismail KMK, Mol BWJ, et al,. TIPPS (Tests in Prediction of Preeclampsia's Severity) Review Group. Accuracy of liver function tests for predicting adverse maternal and fetal outcomes in women with preeclampsia: a systematic review. Acta

Obstetrician

etGynecologicaScandinavica2011; 90: 574-85.

23. Dong X, Gou W, Li C, Wu M, Han Z, Li X, et al,. Proteinuria in preeclampsia: Not essential to diagnosis but related to disease severity and fetal outcomes. Pregnancy hypertension2017; 8: 60-64.

24. Chung WH and To WWK. Outcome of pregnancy with new onset proteinuria and progression to pre-eclampsia: A retrospective analysis. Pregnancy hypertension2018; 12: 174177.

25. Gunel T, Zeybek YG, Akçakaya P, Kalelioğlu I, Benian A, Ermis H, et al,. Serum microRNA expression in pregnancies with preeclampsia.Genetics and Molecular Research2011; 10: 4034-4040.
26. Anton L, Olarerin-George AO, Schwartz N, Srinivas S, Bastek J, Hogenesch JB, et al,. MiR210 inhibits trophoblast invasion and is a serum biomarker for preeclampsia. American Journal of Pathology2013; 183: 1437-1445.

27. Li Q, Long A, Jiang L, Cai L, Xie L, Gu J et al. Quantification of preeclampsia-related microRNAs in maternal serum. Biomedical reports. 2015;3:792-796.

28. Nejad RMA, Saeidi K, Gharbi S, Salari Z and Saleh-Gohari N. Quantification of circulating miR-517c-3p and miR-210-3p levels in preeclampsia. Pregnancy Hypertension2019; 16: $75-78$.

29. Zhang Y, Fei M, Xue G, Zhou Q, Jia Y, Li L, et al,. Elevated levels of hypoxia-inducible microRNA-210 in pre-eclampsia: New insights into molecular mechanisms for the disease. Journal of Cellular and Molecular Medicine2012; 16: 249259.

30. Li J, Wu G, Cao Y and Hou Z. Roles of miR-210 in the pathogenesis of preeclampsia.Archives of Medical Science2019; 15: $183-190$

31. Enquobahrie DA, Abetew DF, Sorensen TK, Willoughby D, Chidambaram K and Williams MA. Placental microRNA expression in pregnancies complicated by preeclampsia. American journal of obstetrics and gynecology2011; 204: 178.e12-21

32. Luo R, Shao X, Xu P, Liu Y, Wang Y, Zhao $\mathrm{Y}$ et al. MicroRNA-210 contributes to preeclampsia by downregulating potassium channel modulatory factor 1. Hypertension2014; 64:839-45. 
33. Munaut C, Tebache L, Blacher S, Noël A, Nisolle $\mathrm{M}$ and Chantraine F. Dysregulated circulating miRNAs in preeclampsia. Biomedical reports. 2016;5(6):686-92 .

34. Muralimanoharan S, Maloyan A, Mele J, Guo C, Myatt LG and Myatt L. MIR-210 modulates mitochondrial respiration in placenta with preeclampsia. Placenta2012; (33):816-23.

35. Luo R, Wang $\mathrm{Y}, \mathrm{Xu} \mathrm{P}, \mathrm{Cao} \mathrm{G}$, Zhao Y, Shao $\mathrm{X}$, et al,. Hypoxia-inducible miR-210 contributes to preeclampsia via targeting thrombospondin type I domain containing 7A. Scientific reports2016; 6: 19588.

36. Luo S, Cao $\mathrm{N}$, Tang $\mathrm{Y}$ and $\mathrm{Gu} \mathrm{W}$. Identification of key microRNAs and genes in preeclampsia by bioinformatics analysis.PLoS ONE2017; 12.

37. Youssef HM and Marei ES. Association of MicroRNA-210 and MicroRNA-155 with severity of preeclampsia. Pregnancy Hypertension2019; 17:49-53.

To cite this article: Fetnat M. Tolba, Adel M. Agha , Maha Rachwan, Basma E. Sakr , Maha A. Abdella, Amira M. Abdelrahman. Evaluation of MicroRNA-210(miR-210) as a Diagnostic and Prognostic Biomarker in Pre-eclampsia Pregnancies, BMFJ 2021; 38(1): 7993. DOI: $10.21608 / \mathrm{bmfi} .2020 .42973 .1317$. 